\title{
A* Algorithm for Shortest Path in Robot Motion
}

\author{
Hameedah Sahib Hasan \\ Ministry of Higher Education and Scientific Research University \\ Al-Furat Al-Awsat Technical University \\ Iraq \\ hameedah211ou@gmail.com
}

*Corresponding Author email: hameedah211ou@gmail.com 


\section{A B S T R A C T}

Mobile robot motion in real-time has many challenges in terms of reaching the exact destination and avoid obstacles. In this work, $A^{*}$ algorithm has been selected to show the robot motion in simulation through Matlab software. Different destinations are selected with several obstacles. A * algorithm shows the ability to achieve the shortest path distance for mobile robot motion as well to avoid different obstacles. Thus, the $A^{*}$ algorithm can be an attractive choice to achieve the best shortest path distance for Mobile robot motion.

Keywords: Mobile Robot, Obstacles, A*Algorithm

\section{Research Objectives}

This paper aims to give an overview of the $A^{*}$ algorithm and to show the robot's motion to avoid different obstacles. $A^{*}$ algorithm is one of the famous algorithms that is used to find the shortest path for robot motion. Several points required to design the algorithms for robot motion, first is to make the motion is easy and feasible as it related to arrival time, the second to avoid static obstacles, third to successfully planned the trajectories of other robots (1). The $A^{*}$ algorithm is based on a heuristic approach. The use of the heuristic variable $\mathrm{H}(\mathrm{s})$ is to assist the algorithm determination. It can achieve better performance by using it to guide its search where the recent node becomes a first selection for searching late (1). Besides, the efficiency of the $A^{*}$ algorithm can improve by ignored the irrelevant

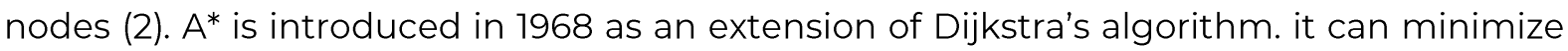
the complexity of calculation compared with Dijkstra's algorithm and can solve the issue related to the increased number of nodes which is affected to the efficiency of Dijkstra's algorithm and led to decreases it's significant (3)(4).

\section{Results}

Robot motion in navigation systems has an important role related to finding minimum travel distance among multi-distentions. To achieve this goal several algorithms are used to find the shortest distance (5). Fig. 1 shows the use of the $A^{*}$ algorithm in free space, the robot motion starts from point $(1,1)$ to multi stations. The total distance achieved is $8.242 \mathrm{~m}$. The robot motion here considers no obstacle. While Fig. 2 shows several obstacles are located at four positions. The robot motion starts from point $(1,1)$ to point $(6,6)$, The robot reaches multi-destination and the total distance is $8.829 \mathrm{~m}$. 


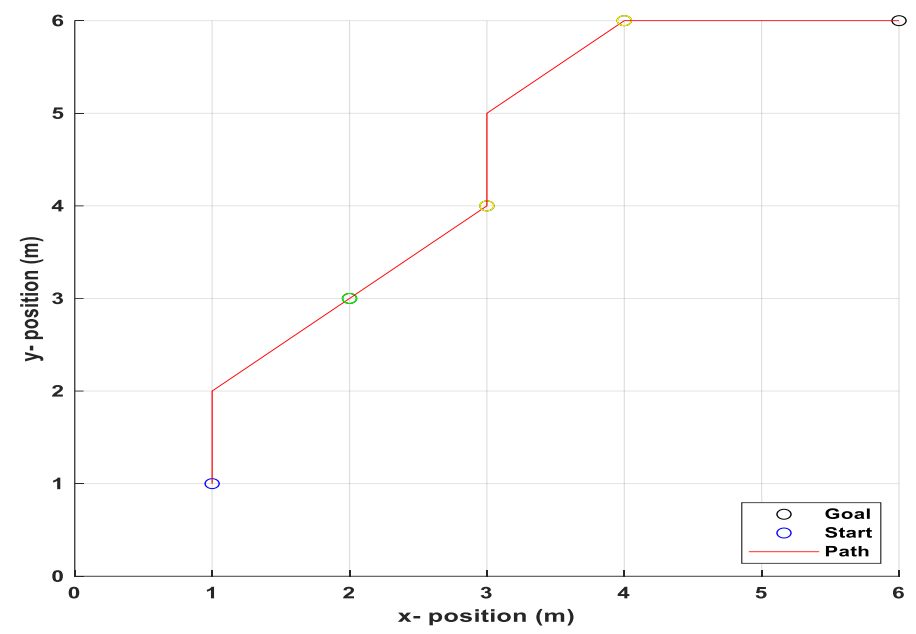

Fig. 1. Mobile robot motion using $A^{*}$ Algorithm with free workspace

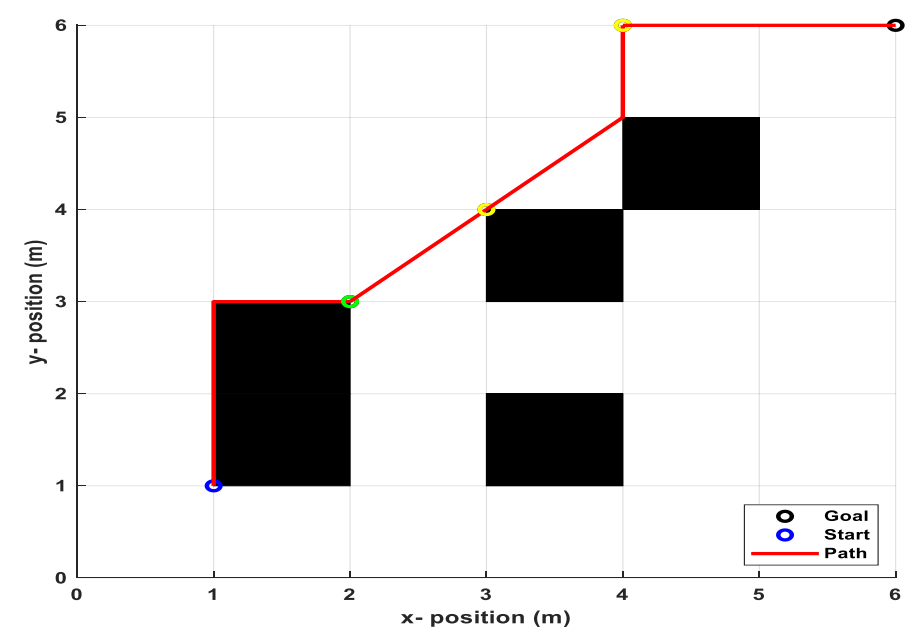

Fig. 2. Mobile robot motion using $A^{*}$ Algorithm with five obstacles

\section{Conclusion}

In this work, the $\mathrm{A}^{*}$ algorithm has been selected to show different robot motions. From the result, the traveling distance obtained through simulation is depending on the obstacles distributed in the traveling area. As well it shows that the $A^{*}$ algorithm choice the path with a minimum traveling distance.

\section{Acknowledgement}

State any acknowledgement if it is required.

\section{References}

1. Hameedah Sahib Hasan, Mohamad Shukri Zainal Abidin, Mohd Saiful Azimi Mahmud MFM. Automated Guided Vehicle Routing: Static, Dynamic and Free range. ternational J Eng Adv Technol. 2019; 8(5): 1-7.

2. Dahari M Bin, Yang L. A Review of Auto-Guided-Vehicles routing algorithms. ICAMMP 2011 Adv Mater. 2011; 1-17. 
3. Jia F, Ren C, Chen Y, Xu Z. A System Control Strategy of a Conflict-free Multi- AGV Routing based on Improved A* Algorithm. 2017 24th Int Conf Mechatronics Mach Vis Pract. 2017; 1-6.

4. Cai K, Wang C, Cheng J, de Silva CW, Meng MQH. Mobile robot path planning in dynamic environments: A survey. arXiv. 2020;

5. Hsu WJ. Scheduling and Routing Algorithms for AGVs: a Survey Scheduling and Routing Algorithms for AGVs: a Survey School of Applied Science Nanyang Technological University. 2014;(June). 